\title{
Modeling the Pedestrian Ability of Detecting Lanes and Lane Changing Behavior
}

\author{
Mohammed Mahmod Shuaib ${ }^{1}$ \\ ${ }^{1}$ Al-Imam Muhammad Ibn Saud Islamic University (IMSIU), Saudi Arabia \\ Correspondence: Mohammed Mahmod Shuaib, College of Shari'a and Islamic Studies in Al Ahsaa, Department \\ of Computer Sciences, Al-Imam Muhammad Ibn Saud Islamic University (IMSIU), Al Ahsaa 31982, Saudi \\ Arabia. E-mail: mh_shuaib@yahoo.com
}

Received: February 20, 2016

Accepted: February 29, 2016

Online Published: March 17, 2016

doi:10.5539/mas.v10n7p1

URL: http://dx.doi.org/10.5539/mas.v10n7p1

The research is financed by (Al-Imam Muhammad Ibn Saud Islamic University under the project (Number 350807))

\begin{abstract}
Incorporating decision-making capability as an intelligence aspect into crowd dynamics models is crucial factor for reproducing realistic pedestrian flow. Crowd dynamics models are still suffering from poor representation of essential behaviors such as lane changing behavior. In this article, we provide the simulated pedestrians in the social force model more intelligence as an extension to the pedestrian's investigation capability in bidirectional walkways, to let the model appear more representative of what actually happens in reality. In the proposed model, the lane's structure is modeled as social network. Thereby, the simulated pedestrians with inconvenient walking can detect the available lanes inside his environment, investigate their attractions, and then make decisions to join the most attractive one. Simulations are performed to validate the work qualitatively by tracing the behavior of the simulated pedestrians and studying the impact of this behavior on lane formation. Finally, a quantitative measurement is used to study the effect of our contribution on the pedestrians' efficiency of motion.
\end{abstract}

Keywords: crowd dynamics, decision making, lane changing behavior, social network

\section{Introduction}

Massive congestion is a challenging problem which has attracted considerable number of researchers to devote much attention to pedestrian dynamics studies to provide solutions. Correcting the pedestrians walking behaviors has been proven to be an essential factor to alleviate the negative consequences of such problem. Researchers have been provoked to improve simulations models to help simulate the aspects of the multidirectional pedestrian flow and correspondingly examine the solutions for better crowd behavior and environmental design. A variety of models has been proposed in microscopic studies to represent the real aspects of the pedestrians walking in order to examine the proposed solutions. Among which are the Social Force Model (Helbing \& Monlar, 1995; Helbing, Farkas, \& Vicsek, 2000 and Shuaib, 2014), Cellular Automata Models (Blue \& Adler, 2000 and Burstedde, Klauck, Schadschneider \& Zittartz, 2001) and the Discrete Choice Models ( Antonini, Bierlaire \& Weber, 2006 and Robin, Antonini, Bierlaire \& Cruz, 2009). The models are categorized as operational level class, which describes the instantaneous behaviors that involve most activities resulting from the interactions among pedestrians (e.g. avoiding collisions, deviations, acceleration and deceleration). For the purpose of validation, the models are essentially required to introduce real aspects of the multidirectional flow behavior such as the self-organization phenomena and reproduce its characteristics accurately as well.

Lane formation, as an interesting self-organization phenomenon, and its effect on the reproduction of bidirectional flow aspects has received much attention from researchers such as (Blue \& Adler, 2000; Burstedde et al., 2001; Nowak et al., 2013; Guo, 2014; Zhang, 2015 \& Guo, Wang and Zheng, 2015). Nowak et al. (2013) proposed swapping, politeness, and anticipation factors to obtain model coincide with experimental data. Such factors present the role of the opposite pedestrians. Spatial separation rules as suggested by Guo (2014) was provided to pedestrians exiting a room to constitute lanes. Guo et al. (2015) discussed two mechanisms leading to the lane formation and they conclude that avoiding oncoming pedestrians is more relevant to the reproduction 
of lane formation than following pedestrians walking in the same direction. Zhang (2015) investigated the guide, press and contain effects performed by pedestrians, with same and opposite directions, on the lane changing decision.

Experimentally, Guo et al. (2012) studied the effect of the look-ahead behavior on the pedestrian bidirectional flow. Zhang et al. (2012) studied the behavior of the opposing flows in a corridor. They concluded that pedestrians' movement is more systematic and the lanes are stable and more coherent when the pedestrians' exits are predetermined. This shows that the importance of the subjective consciousness of the tactical level of behaviors. Researchers such as Hoogendoorn and Bovy (2004); Daamen (2004); and Asano, Iryo and Kuwahara (2010) indicated the significance of obtaining integrated models comprised of two complementary levels: the operational and the tactical levels. It is worth mentioning that detecting far lanes and joining them as a tactical level behavior have not been studied in the aforementioned studies. Such behavior is commonly followed by pedestrians to alleviate their inconvenient walking, and accordingly this behavior is essential factor for presenting a well-representative model that performs the decision making capability with the consideration of lane-changing capability.

In this article, we propose a tactical model to be incorporated into the Social Force Model. The proposed model is based on the assumption that the pedestrian who walks inconveniently, because of the opposite flow, can detect lanes in order to join, to relieve his inconvenience. This ability is represented by the pedestrian investigating his current situation within his sights and then making a short-term decision. This paper is organized as follows. In the next section we introduce the Social Force Model and the decision making capabilities incorporated into the model. In the third section, a model of detecting lanes and joining the attractive one are introduced. Finally, simulations to establish the results of our work are conducted.

\section{Social Force Model and Decision Making Capability}

The Social Force Model (SFM) is a continuous microscopic model developed by Helbing and Molnar (1995). The model is characterized with its representation of pedestrian's motivations in terms of the surrounding pedestrians and obstacles as social forces. The sum of these forces is implemented in a semi-Newtonian equation, resulting in the acceleration of the pedestrian's movement. Helbing et al. (2000) incorporated into the model physical forces which arise in the case of physical interactions amongst the pedestrians. The main equations of the model are:

$$
\begin{gathered}
\frac{d \vec{x}_{i}(t)}{d t}=\vec{v}_{i}(t), \\
m_{i} \frac{d \vec{v}_{i}(t)}{d t}=\vec{f}_{i}^{\text {pref }}(t)+\sum_{j} \vec{f}_{i j}(t)+\sum_{k} \vec{f}_{i, \text { object }, k}(t)+\varepsilon_{i}(t), \\
\vec{f}_{i}^{\text {pref }}(t)=\gamma_{i}\left(v_{i}^{0}(t) \vec{e}_{i}^{0}(t)-\vec{v}_{i}(t)\right),
\end{gathered}
$$

where $\frac{d \vec{x}_{i}(t)}{d t}$ is the rate of change in the location of pedestrian $i$ at time $t ; \frac{d \vec{v}_{i}}{d t}(t)$ is the acceleration of pedestrian $i$ resulting from the sum of the total forces upon him; $\varepsilon_{i}(t)$ is the fluctuation of pedestrian $i$; the function $\vec{f}_{i}^{\text {pref }}(t)$ is the preferred force modeled to express the motivation of the pedestrian $i$ to adapt his actual velocity $\vec{v}_{i}(t)$ to reach another velocity $v_{i}^{0}(t) \vec{e}^{0}(t)$ at which he prefers to walk at time $t$, where $v_{i}^{0}(t)$ is the preferred speed and $\vec{e}^{0}(t)$ is the preferred direction; and the function $\vec{f}_{i j}(t)$ and $\vec{f}_{i, \text { object, } k}(t)$ are the sum of all forces exerted by pedestrian $j$ and object, $k$ upon pedestrian $i$. All activities resulting from the equations (1-3) such as acceleration, deceleration, and avoiding collisions are examples of operational levels based on SFM. For unidirectional walkway, Zainuddin and Shuaib (2010) and Shuaib, Alia and Zainuddin (2013) incorporated 
decision-making capabilities as tactical level behavior, where they proposed the ZS model to be incorporated into the SFM to enable the simulated pedestrians make decisions to avoid semi-blocked situations such as obstacles and grouped pedestrians. The model was represented by determining intermediate points for their walking, accordingly changing their routes, and consequently overtaking the encountered blocked situations. The (ZS) model has been modified in (Shuaib \& Zainuddin, 2015) to involve aspects of bidirectional flow and its effects on lane formation. The model was validated by reproducing lanes compatible to the lane formation in the experimental study (Zhang et al, 2012).

\section{Modeling Lane's Structure and Lane Joining}

\subsection{Modeling Lane's Structure}

With the assumption of uniform physical appearance of pedestrians, pedestrian $i$ who is walking inconveniently can detect pedestrian gathering structures based on specific collective behavior of pedestrians such as closeness and velocities. We propose the structure as a network, where the nodes are the pedestrians who constitute the structures, and where the connections of these nodes are determined by closeness and velocities. We assume first that all pedestrians are classified into two opposite-moving structures. A pedestrian $l$ with considerably different velocity from the average velocity of his structure could form a separate self-organized and dynamic structure if he is surrounding by others walking with similar velocity. This possible structure is determined as follows: Firstly, we form the objective connection set, $\mathrm{Ob}_{-}$set $_{l}$, of pedestrian $l$ by connecting pedestrian $l$ to the immediately other surrounding pedestrians. Secondly, we form the subjective connection set, $S_{-}$set $t_{l}$ by excluding those who are located at far positions and walking at unusual velocity, compared with the surrounding pedestrians. We assign the following value ( 0 or 1$)$ to each connection:

$$
\begin{aligned}
& \text { connect }_{l j}=\delta_{A_{l}}(j) * \delta_{B_{l}}(j), \\
& A_{l}=\left\{k \in O b_{-} \operatorname{Set}_{l}|\quad| v_{l}-\vec{v}_{k} \cdot \vec{e}_{l} \mid \leq a \sigma_{\text {Ob_s }_{-} \text {set }_{l}}^{v}\right\} \\
& B_{l}=\left\{k \in O b_{-} s_{l} e t_{l}|| d i s t_{l j}-M_{O b_{-} \text {set }_{l}}^{\text {dist }} \mid \leq M_{\text {Ob_- }_{\text {set }}}^{\text {dist }}+b \sigma_{O b_{-} \text {set }_{l}}^{\text {dist }}\right\}
\end{aligned}
$$

where $\sigma_{O b_{-} s e t_{l}}^{v}$ is the standard deviation of pedestrians' velocities in $O b_{-} \operatorname{set}_{l} ; M_{O b_{-} \text {set }_{l}}^{\text {dist }} \sigma_{O b_{-} s t_{l}}^{\text {dist }_{1}}$ are the mean and standard deviation of pedestrians' distances from pedestrian $l$ in $O b_{-} \operatorname{set}_{l}$; and $a$ and $b$ are constant parameters. The function $\delta_{A_{l}}(j)$ is essential factor to exclude the pedestrians whose velocities do not match the velocity of pedestrian $l$, at least $a \sigma_{O b_{-} s e t_{l}}^{v}$. Similarly, the function $\delta_{B_{l}}(j)$ is essential factor to exclude pedestrians located far than the other immediate adjacent pedestrians. However, its role is not crucial in extreme global densities in unidirectional or multi-directional walkways. The subjective connection set for pedestrian $l$ is as follows:

$$
S_{-} \text {set }_{l}=\left\{j \mid \text { connect }_{l j}=1\right\} .
$$

It is worth mentioning here that the connection of pedestrian $l$ with pedestrian $j$ does not mean that 
$S_{-}$set $_{l}=S_{-}$set $_{j}$. We introduce Structure $_{l}$ notion to denote the set of all pedestrians connected to pedestrian $l$ with a path (list of connections). Finally, using equivalence classes created by connection relation,

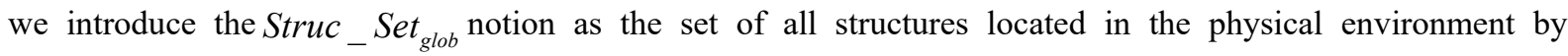
imposing the following: Structure $_{l}=$ Structure $_{j}$ if there is a path between pedestrian $l$ and $j$, and Structure $_{l} \cap$ Structure $_{j}=\phi$ if no path between them. For pedestrian $i$, Struc $_{-}$Set $_{i}$ notion is introduced to denotes the set of all structures located within his sight.

In this article, we focus on introducing lanes, and accordingly we compose the subjective set of the lanes Lanes_Set $t_{i}$ for pedestrian $i$ with the consideration of all structures in Struc $\_S_{i} t_{i}$ that have significant sizes, could be fitted by a linear curve with acceptable error and are directed toward the destination of pedestrian $i$ with acceptable deviation.

\subsection{Modeling the Attractions of Lanes}

The goal of modeling the attraction (repulsion) of lanes under investigation is to let each excited pedestrian join the lane that has the greatest attraction (the least repulsion). We assume that the attraction (repulsion) of a lane is influenced by the specific attributes of two factors: the gap between the pedestrian and the lane and the lane itself. The effect of the gap is associated with its length and the anticipated pedestrians' collisions while penetrating the gap to join the considered lane. The effect of the lane is associated with its average velocity. Thus, we suggest the time, which implicitly includes both velocity and distance, to express the repulsion determined by each factor.

Corresponding to each lane $k$ located within the investigation area of pedestrian $i$, we attach for pedestrian $i$ a route $k$ divided into two parts (e.g. see Figure 2): The gap's part represented by a line that connects pedestrian $i$ to the nearest pedestrian who belongs to lane $k$ and the lane part represented by the fitting line that starts from the point of intersection with the gap's part and ends at the boarder of the investigation area. Besides, we attach for each pedestrian an original route directed to his destination. The last route is considered as the lane part and the gap part has length equals zero.

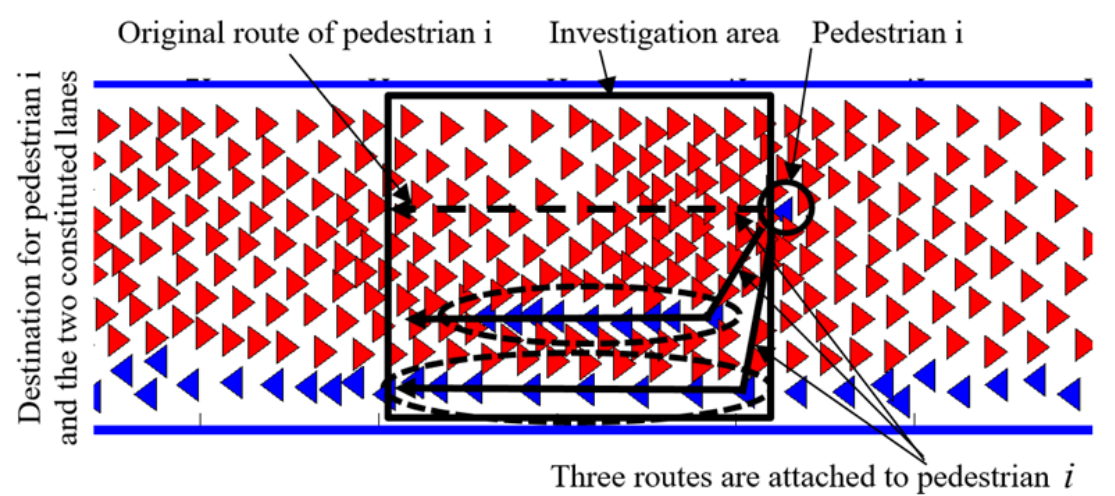

Figure 1. Three routes are attached to pedestrian $i$ : two of them are associated with the constituted lanes moving towards his direction, and the third is the route towards his destination

The lane and gap parts inherit the characteristics of the lane and the associated gap, respectively. The model of the repulsion of route $k$, denoted by Time route $_{k}$, is a linear combination of the repulsive effects of its parts, Time $^{\text {gap }_{k}}$ and Time $^{\text {Iane }_{k}}$, as follows: 


$$
\begin{gathered}
\operatorname{Time}_{i}^{\text {route }_{k, i}}(t)=\operatorname{Time}_{i}^{\text {gap }_{k, i}}(t)+\operatorname{Time}_{i}^{\text {Iane }_{k, i}}(t), \\
\operatorname{Time}_{i}^{\text {gap }_{k, i}}(t)=\frac{\operatorname{length}_{i}^{\text {gap }_{k, i}}(t)}{\vec{v}_{\text {xxper }}\left(\sigma^{\text {gap }_{k, i}}(t)\right)} \\
\operatorname{Time}_{i}^{\text {lane }_{k, i}}=\frac{\text { length }_{i}^{\text {lane }_{k, i}}(t)}{\left\langle\vec{v}_{j}(t)\right\rangle_{j \in \text { lane }_{k}}}
\end{gathered}
$$

where the variable $\sigma^{g a p_{k}}$ is the average local density of the pedestrians in contact with the gap line; $\vec{v}_{E x p e r}\left(\sigma^{g a p_{k, i}}\right)$ is the experimental velocity obtained from experimental data of multidirectional walkway. As can be seen, the greater the lane's velocity the lesser the repulsion effect, and accordingly the more likely the excited pedestrian will choose lane $j$ to follow. On the other hand, higher value of gap density results in lower value of gap velocity and accordingly greater repulsion effect.

The index of the best route assessed by pedestrian $i$ is determined as follows:

$$
\text { BestRoute } \left._{i}(t)=\text { index }_{\left\{\min _{k}\right.}\left(\text { Time }_{i}^{\text {route }_{k, i}}(t)\right) \mid \text { Lane }_{k} \in \text { lane }_{-} \text {set }_{i}\right\} .
$$

Finally, we assign to each pedestrian a variable $D S$ by which his decision should be governed. This decision variable $D S$ is modeled as a function of the variables resulting from the investigation process (the repulsions of the alternative routes):

$$
D S_{i}(t)=\left\{\begin{array}{cc}
1 & \text { Time }_{i}^{\text {CurrRoute }, i}(t)-\text { Time }_{i}^{\text {BestRoute }, i}(t) \geq U_{i} \\
0 & \text { otherwise }
\end{array},\right.
$$

where Time $_{i}^{\text {BestRoute }, i}$ is the repulsion of the new route chosen by pedestrian $i$; Time $e_{i}^{\text {CurrRoute, } i}$ is the repulsion of the current choice at the time of decision; $U_{i}^{\text {th }}$ is the threshold of utility for which the pedestrian $i$ may change his current choice to the new one. The criterion for modeling $U_{i}^{\text {th }}$ is the varying effect of the social attribute among the individuals in the population. High social attribute of a pedestrian renders him more respectful of the others. The member intends to deviate from his current route; it may cause more interactions with other pedestrians or the transgression of community rules such as violating the territorial areas of the others. If he has a low value of his social attribute the value of $U^{\text {th }}$ would decrease such that the pedestrian makes this decision faster than one who has a higher value of his social attribute. Finally, Equation (3) and the desired direction are replaced by

$$
\begin{aligned}
& \vec{f}_{i}^{\text {pref }}(t)=\gamma_{i}\left(v_{i}^{0}(t) \vec{e}_{i}^{\text {CurrRoute }}(t)-\vec{v}_{i}(t)\right), \\
& \vec{e}_{i}^{\text {CurrRoute }}= \begin{cases}\vec{e}_{i}^{\text {BestRoute }} & D S_{\text {change }, i}=1 \\
\vec{e}_{i}^{\text {CurrRoute }} & D S_{\text {change }, i}=0 .\end{cases}
\end{aligned}
$$

\section{Simulation Results and Discussion}

\subsection{Simulation Specification}

To perform simulations, we used MATLAB version 10 on a $2.5 \mathrm{GHz}$ Intel (R) Core i7 workstation. Equations (1) and (2) are solved explicitly by numerical integration (Euler's method). The simulations in the first scenario 
were conducted to exhibit the ability of simulated pedestrians to detect the existence of lanes and joining them for convenient walking. The walkway of the current simulations is straight, with elliptical functionality as in a racetrack (Zainuddin and Shuaib, 2010). The specification of the simulated pedestrians is as follows: The simulated pedestrians are divided into two groups. The positions of the first group were initialized randomly in the simulated area (walkway) simultaneously (see Fig. 2), and their motion instantaneously were directed towards the right (the destination). The second group of pedestrians was directed to the opposite side. Sum pedestrians of the second group were initialized in the upper right part of the walkway. All pedestrians have preferred velocities with the mean equal to $1.34 \mathrm{~m} / \mathrm{s}$ and standard deviation of $0.26 \mathrm{~m} / \mathrm{s}$.

Table 1. Pedestrian parameters

\begin{tabular}{lll}
\hline Variable & Parameter & Simulation value \\
\hline $\mathrm{m}$ & Range of pedestrians' mass & {$[77-83] \mathrm{kg}$} \\
$\mathrm{r}$ & Range of pedestrians' radius & {$[0.25-0.27] \mathrm{m}$} \\
$\varepsilon$ & Range of fluctuation source of the pedestrian's & {$\left[\begin{array}{l}\left.0,(0.1) * v^{0}\right] \\
\end{array}\right.$} \\
& acceleration, which is randomly assigned to each & \\
& individual. & \\
\hline
\end{tabular}

\subsection{Detecting Lanes and Lane Changing Behavior}

The criterion to validate the above work is the appearance of changing routes, that is, the simulated pedestrians from the second group and who are walking in inconvenient routes would change routes to more appropriate ones. In view of this criterion, the simulated pedestrians who are traced are some pedestrians initialized unevenly who change their direction toward the formed lanes to conserve their velocity and walk comfortably.

With long range dimension of the investigation area, the inconveniently walking simulated pedestrians initialized in the right upper part were able to detect the far lane and make a decision to join this lane. While crossing the counter flow, small lanes were constituted. The moderate dense crowd is a reason helped raise the formation of small lanes, while in high dense crowd the pedestrians are forced to be dispersed because of the intense of the head-of conflicts (Guo et al., 2015).

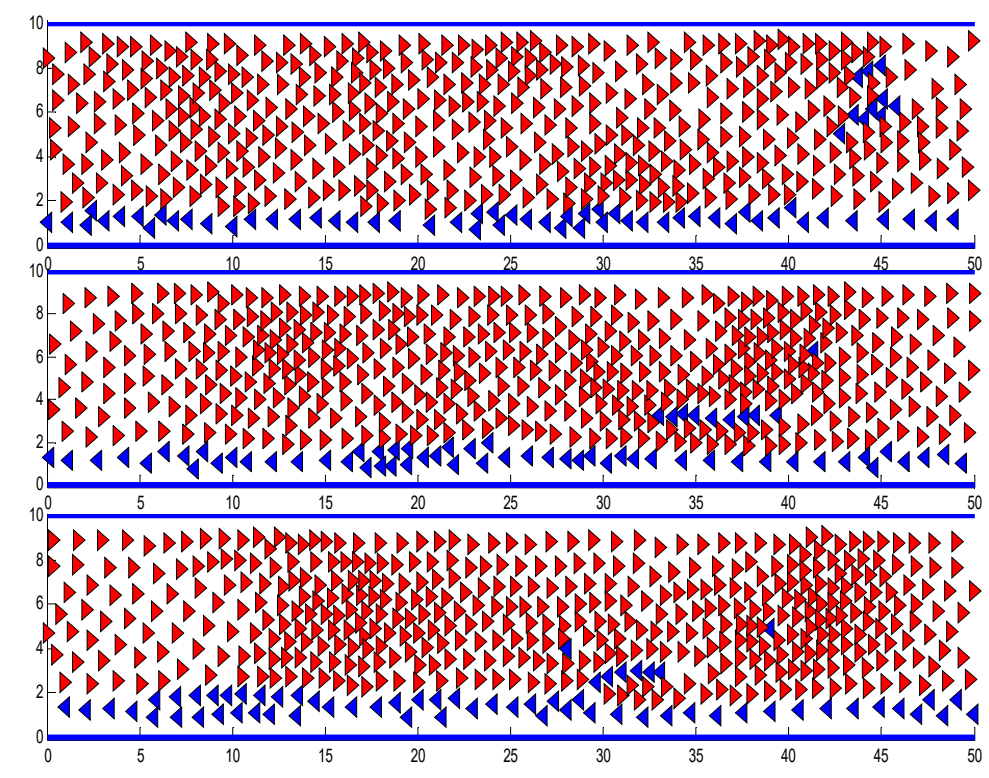

Figure 2. joining lane behavior by group of pedestrians in bidirectional pedestrian flow

\subsection{The Efficiency of Motion}

Three groups of simulations are conducted to study the influence of diverse intersecting angles chosen by the 
pedestrians joining a lane. We define the intersecting angle between two pedestrian flows to be equal to zero if both flows are directed oppositely and to $\pi$ if both flows in the same direction. For each group of simulations, different intersecting angles are considered, that is $0, \pi / 6$ and $\pi / 4$ respectively, and for each angle five simulations are conducted to determine the means and the deviations of the efficiency of pedestrians' motion. The efficiency of motion is a quantitative measurement used by Helbing and Molnar (2001), on the basis of the Social Force Model. This measurement helps optimize the pedestrian facilities, whether the pedestrians are walking conveniently in the considered facility or not.

In Fig. 3, the following results are produced. The resulting mean of the efficiency of motion shown in Fig. 3 slightly decreases with the decrease of the angle of changing the route of motion. The reason for this is that the pedestrians with small intersecting angle are causing less head on conflict than the larger intersecting angle, and accordingly passing each other with less collision. On the contrary, with high angle of intersection, the crossing behavior is the dominant and high contact occurs which delay the motion of the pedestrians. Finally, accounting for the deviation of efficiency of motion for each angle of intersection, it is observed that the deviation when high angle of changing route is higher than the others with lower angles. This is due to the variability of collision occurrence in the high angles simulations. The result indicates that head-on conflicts of different intersecting angles have different influences on the efficiency of motion in the intersecting area. This result urges the necessity of correcting the crossing behavior of the pedestrians by guiding them to the right angle of intersecting with the least effort.

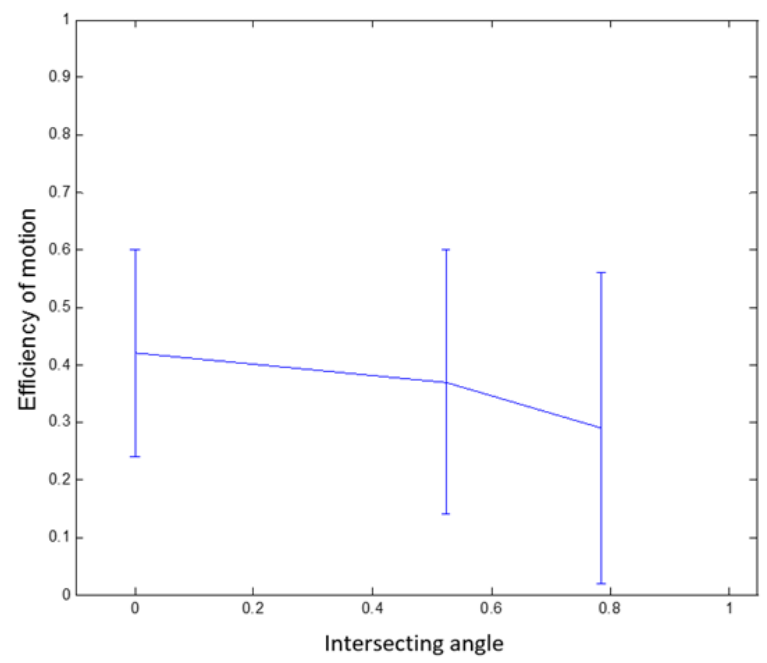

Figure 3. the error bars show the means and deviations of the efficiency of motion with respect to the intersecting angle

\section{Conclusion}

In this work, an intelligence aspect of pedestrian behavior has been integrated into the Social Force Model as a decision-making capability. The pedestrians investigate the area under their sights to look for more comfortable flow to walk in or lanes to join. The simulations have shown the pedestrian's ability of making decisions to join lanes enables the excited pedestrians to reduce the intense of the head-of conflicts resulting from the counter flow, and reduce the potential emergence of blocked situations. Our work results in more efficient motion than the original model. We suggest an extension of this work to involve modeling and detecting various pedestrians' structures, such as groups. Moreover, we encourage investigating their effects on flow by considering different ordering of directional flow and incorporating some aspects such as a semi-blocked situation or physical characteristics such as obstacles and walls.

\section{Acknowledgments}

We thank the Al-Imam Muhammad Ibn Saud Islamic University for supporting this work under the project (Number 350807).

\section{References}

Antonini, G., Bierlaire, M., \& Weber, M. (2006). Discrete choice models of pedestrian walking behavior. 
Transportation Research Part B, 40, 667-687. http://dx.doi.org/10.1016/j.trb.2005.09.006

Asano, M., Iryo, T., \& Kuwahara, M. (2010). Microscopic pedestrian simulation model combined with a tactical model for route choice behavior. Transportation Research Part C, 18, 842-855. http://dx.doi.org/10.1016/j.trc.2010.01.005

Blue, V., \& Adler, J. (2000). Cellular automata microsimulation of bi-directional pedestrian flows. Journal of the Transportation Research Board, 1678, 135-141. http://dx.doi.org/10.3141/1678-17

Burstedde, C., Klauck, K., Schadschneider, A., \& Zittartz, J. (2001). Simulation of pedestrian dynamics using a $\begin{array}{lllll}\text { two-dimensional cellular automaton. Physica } & \text { A, 295, }\end{array}$ http://dx.doi.org/10.1016/S0378-4371(01)00141-8

Daamen, W. (2004). Modelling Passenger Flows in Public Transport Facilities. Ph. D. Thesis, Delft University of Technology, Netherlands.

Guo, R.Y., Wong, S. C., Xia, Y. H., Huang, H. J., Lam, W. H. K., \& Choi, K. (2012). Empirical evidence for the look-ahead behavior of pedestrians in bi-directional flows. Chin. Phys. Lett., 29(6), 1-4. http://dx.doi.org/10.1088/0256-307X/29/6/068901

Guo, R. Y. (2014). Simulation of spatial and temporal separation of pedestrian counter flow through a bottleneck Physica A 415 428. http://dx.doi.org/10.1016/j.physa.2014.08.036

Guo, W., Wang X., \& Zheng X. (2015). Lane formation in pedestrian counter flows driven by a potential field considering following and avoidance behaviors, Physica A., 431 87-101.

Helbing, D., \& Molnár, P., (1995). Social Force Model for pedestrian dynamics. Physical Review E., 51, 4282-7. http://dx.doi.org/10.1103/PhysRevE.51.4282

Helbing, D., Farkas, I., \& Vicsek, T. (2000). Simulating dynamical features of escape panic. Nature, 407, 487-90. http://dx.doi.org/10.1038/35035023

Helbing, D., Buzna, L., Johansson, A., \& Werner, T. (2005). Self-Organized Pedestrian Crowd Dynamics: Experiments, Simulations, and Design Solutions, Transp. Sci., 39, 1-24.

Helbing, D., Farkas, I. J., \& Vicsek, T. (2001). Freezing by heating in a driven mesoscopic system, Phys. Rev. Lett. 84, 1240. http://dx.doi.org/10.1103/PhysRevLett.84.1240

Hoogendoorn, S. P., \& Bovy, PHL. (2004) Pedestrian route-choice and activity scheduling theory and models. $\begin{array}{lllll}\text { Transportation Research Part B: } & \text { Methodological, } & \text { 38(2), } & \text { 169-190. }\end{array}$ http://dx.doi.org/10.1016/S0191-2615(03)00007-9

Lian, L., Mai, X., Song, W., Richard, Y., Wei, X., \& Ma, J. (2015). J. Stat. Mech. P08024. http://dx.doi.org/10.1088/1742-5468/2015/08/P08024

Nowak, S., \& Schadschneider, A. (2013). A Cellular Automaton Approach for Lane Formation in Pedestrian Counter flow, Traffic and Granular Flow, 11,149-160. http://dx.doi.org/10.1007/978-3-642-39669-4_15

Robin, T., Antonini, G., Bierlaire, M., \& Cruz, J. (2009). Specification, estimation and validation of a pedestrian walking behavior model. Transportation Research Part B: Methodological, 43(1), 36. http://dx.doi.org/10.1016/j.trb.2008.06.010

Shuaib, M. M., Alia, O. M., \& Zarita, Z. (2013). Incorporating prediction factor into the investigation capability in the social force model: application on avoiding grouped pedestrians, Appl. Math. Inf. Sci., 7, 323.

Shuaib, M. M. (2014). Preserving Socially Expected Crowd Density in front of the Exit for the Reproduction of Experimental Data by Modeling the Pedestrians' behind Perception. J. Stat. Mech., P10037. http://dx.doi.org/10.1088/1742-5468/2014/10/P10037

Shuaib, M. M., \& Zainuddin, Z. (2015) An investigation capability model for bidirectional pedestrian flow, modern Applied Science, 9, 12. http://dx.doi.org/10.5539/mas.v9n12p88

Zainuddin, Z., \& Shuaib, M. (2010). Incorporating Decision Making Capability into the Social Force Model in Unidirectional Flow. Research Journal of Applied Sciences, 5(6), 388-393. http://dx.doi.org/10.3923/rjasci.2010.388.393

Zhang, J., Klingsch, W., Schadschneider, A., \& Seyfried, A. (2012). Ordering in bidirectional pedestrian flows and its influence on the fundamental diagram, J. Stat. Mech., 02002. http://dx.doi.org/10.1088/1742-5468/2012/02/P02002 
Zhang, Q. (2015). Simulation model of bi-directional pedestrian considering potential effect ahead and behind. Physica A 419 335-348. http://dx.doi.org/10.1016/j.physa.2014.09.054

\section{Copyrights}

Copyright for this article is retained by the author(s), with first publication rights granted to the journal.

This is an open-access article distributed under the terms and conditions of the Creative Commons Attribution license (http://creativecommons.org/licenses/by/3.0/). 